\title{
A criterion for a monomial ideal to have a linear resolution in characteristic 2
}

\author{
E. Connon* \\ Department of Mathematics \& Statistics \\ Dalhousie University \\ Halifax NS B3H 4R2, Canada \\ emmaconnon@hotmail.com
}

\author{
Sara Faridi ${ }^{\dagger}$ \\ Department of Mathematics \& Statistics \\ Dalhousie University \\ Halifax NS B3H 4R2, Canada \\ faridi@dal.ca
}

Submitted: Feb 2, 2014; Accepted: Feb 25, 2015; Published: Mar 13, 2015

Mathematics Subject Classifications: 05E40, 13D02, 13F55

\begin{abstract}
In this paper we give a necessary and sufficient combinatorial condition for a monomial ideal to have a linear resolution over fields of characteristic 2 .

Keywords: linear resolution, monomial ideal, chordal graph, simplicial complex, simplicial homology, Stanley-Reisner complex, edge ideal, facet ideal, chorded complex, chordal hypergraph, Fröberg's theorem
\end{abstract}

\section{Introduction}

Recently there has been interest in finding a characterization of square-free monomial ideals with linear resolutions in terms of the combinatorics of their associated simplicial complexes or hypergraphs. See, for example, [5], [8], [9], [10], and [11]. This exploration was motivated by a theorem of Fröberg from [7] in which he gives the following combinatorial classification of the square-free monomial ideals generated in degree two which have linear resolutions.

Theorem 1 (Fröberg [7]). The edge ideal of a graph $G$ has a linear resolution if and only if the complement of $G$ is chordal.

This characterization has inspired the introduction of several different definitions of a "chordal" hypergraph with the goal of achieving a generalization of Fröberg's theorem to higher-dimensions. Emtander [5] and Woodroofe [11] use their respective definitions

${ }^{*}$ Research supported by a Killam scholarship.

${ }^{\dagger}$ Research supported by NSERC. 
of a "chordal" hypergraph to give a sufficient condition for a square-free monomial ideal to have a linear resolution over all fields. In [3], the authors introduce the notion of a $\boldsymbol{d}$-chorded simplicial complex and use it to give a necessary combinatorial condition for an ideal to have a linear resolution over all fields.

Obtaining a complete generalization of Fröberg's theorem to higher dimensions is made difficult by the fact that there exist square-free monomial ideals which have linear resolutions over some fields and not others. In particular the existence of a linear resolution depends on the characteristic of the field. The Stanley-Reisner ideal of the triangulation of the real projective plane is a typical example and has a linear resolution only over fields of characteristic not equal to 2. Such examples tell us that when an ideal is generated in degrees higher than two it is not always the combinatorics of the associated simplicial complex that determines the existence of a linear resolution. In this paper we concentrate on fields of characteristic 2 because in this case we have a more direct relationship between the combinatorics of a complex and its simplicial homology (see [2]) which is of primary interest when determining the existence of a linear resolution.

The condition given in [3] is not sufficient to ensure linear resolution. In this paper we are able to characterize the obstructions to the converse over fields of characteristic 2 by demonstrating that all counter-examples share a specific combinatorial property. In Section 3 we are able to give the following necessary and sufficient condition for an ideal to have a linear resolution over fields of characteristic 2 based on the combinatorial structure of the Stanley-Reisner complex of the ideal.

Theorem 2. Let I be generated by square-free monomials in the same degree. Then I has a linear resolution over fields of characteristic 2 if and only if the Stanley-Reisner complex of I is chorded.

Using this result one can give a new combinatorial proof of Theorem 1 over fields of characteristic 2 (Connon [1]).

\section{Background}

Let $k$ be a field and let $R=k\left[x_{1}, \ldots, x_{n}\right]$. For any monomial ideal $I$ in $R$ there is a minimal graded free resolution of $I$ of the form

$$
0 \rightarrow \bigoplus_{j} R(-j)^{\beta_{m, j}(I)} \rightarrow \bigoplus_{j} R(-j)^{\beta_{m-1, j}(I)} \rightarrow \cdots \rightarrow \bigoplus_{j} R(-j)^{\beta_{0, j}(I)} \rightarrow I \rightarrow 0
$$

where $R(-j)$ denotes the free $R$-module obtained by shifting the degrees of $R$ by $j$ and $m \leqslant n$. The numbers $\beta_{i, j}(I)$ are called the graded Betti numbers of $I$. We say that $I$ has a $\boldsymbol{d}$-linear resolution over $\boldsymbol{k}$ if $\beta_{i, j}(I)=0$ for all $j \neq i+d$. It follows that $I$ is generated in degree $d$.

It is known that classifying monomial ideals with linear resolutions is equivalent to classifying Cohen-Macaulay monomial ideals and that it is sufficient to consider squarefree monomials $[4,6]$. 
By studying square-free monomial ideals we are able to make use of techniques from Stanley-Reisner theory and facet ideal theory by associating our ideal to a combinatorial object. Recall that an (abstract) simplicial complex $\Gamma$ on the finite set of vertices $V(\Gamma)$ is a collection of subsets of $V(\Gamma)$ called faces or simplices such that if $F \in \Gamma$ and $F^{\prime} \subseteq F$ then $F^{\prime} \in \Gamma$. The faces of $\Gamma$ that are not strictly contained in any other face of $\Gamma$ are called facets and we denote the facet set of $\Gamma$ by $\operatorname{Facets}(\Gamma)$. If $\operatorname{Facets}(\Gamma)=\left\{F_{1}, \ldots, F_{k}\right\}$ then we write

$$
\Gamma=\left\langle F_{1}, \ldots, F_{k}\right\rangle .
$$

The dimension of a face $F$ of $\Gamma$ is equal to $|F|-1$. A face of $\Gamma$ of dimension $d$ is referred to as a $\boldsymbol{d}$-face. The dimension of the simplicial complex $\Gamma$, denoted by $\operatorname{dim} \Gamma$, is the maximum dimension of its facets. The complex $\Gamma$ is pure if these facets all share the same dimension.

The pure $\boldsymbol{d}$-skeleton of a simplicial complex $\Gamma$, written $\Gamma^{[d]}$, is the simplicial complex whose facets are the faces of $\Gamma$ of dimension $d$. The complex $\Gamma$ is said to be $\boldsymbol{d}$-complete when all $(d+1)$-subsets of $V(\Gamma)$ are faces of $\Gamma$. The $d$-complete complex on $n$ vertices is denoted $\Lambda_{n}^{d}$. The $\boldsymbol{d}$-complement of $\Gamma$ is the complex $\bar{\Gamma}_{d}$ with

$$
\operatorname{Facets}\left(\bar{\Gamma}_{d}\right)=\{F \subset V(\Gamma)|| F \mid=d+1, F \notin \Gamma\} .
$$

The induced subcomplex of $\Gamma$ on the vertex set $S \subseteq V(\Gamma)$, denoted $\Gamma_{S}$, is the simplicial complex whose faces are those faces of $\Gamma$ contained in $S$.

A pure $d$-dimensional simplicial complex is $\boldsymbol{d}$-path-connected when each pair of $d$ dimensional faces are joined by a sequence of $d$-dimensional faces where adjacent pairs intersect in $(d-1)$-faces. The $\boldsymbol{d}$-path-connected components of a pure $d$-dimensional simplicial complex are the maximal subcomplexes which are $d$-path-connected.

The Stanley-Reisner complex of the square-free monomial ideal $I$ in the polynomial ring $k\left[x_{1}, \ldots, x_{n}\right]$ is the simplicial complex on the vertices $x_{1}, \ldots, x_{n}$ whose faces are given by the square-free monomials not belonging to $I$. The Stanley-Reisner complex of $I$ is denoted $\mathcal{N}(I)$. Conversely, the Stanley-Reisner ideal of the simplicial complex $\Gamma$, denoted $\mathcal{N}(\Gamma)$, is the ideal generated by monomials $x_{i_{1}} x_{i_{2}} \cdots x_{i_{k}}$ such that $\left\{x_{i_{1}}, x_{i_{2}}, \ldots, x_{i_{k}}\right\}$ is not a face of $\Gamma$. See Figure 1 for an example of this relationship.

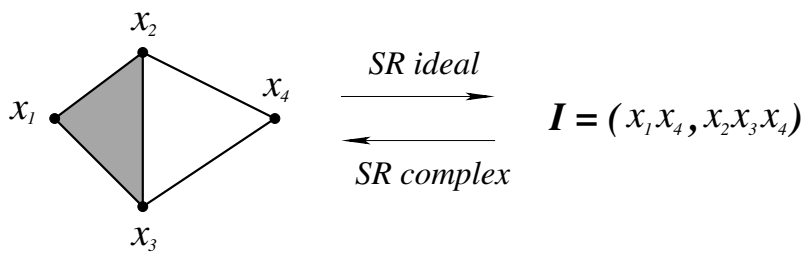

Figure 1: Stanley-Reisner relationship

The facet complex of the square-free monomial ideal $I$ in $k\left[x_{1}, \ldots, x_{n}\right]$ is the simplicial complex $\mathcal{F}(I)$ on the vertices $x_{1}, \ldots, x_{n}$ whose facets are given by the minimal 
monomial generators of $I$. The facet ideal of the complex $\Gamma$ is generated by the monomials $x_{i_{1}} x_{i_{2}} \cdots x_{i_{k}}$ such that $\left\{x_{i_{1}}, x_{i_{2}}, \cdots, x_{i_{k}}\right\}$ is a facet of $\Gamma$. The facet ideal of $\Gamma$ is denoted by $\mathcal{F}(\Gamma)$. An example is given in Figure 2 .

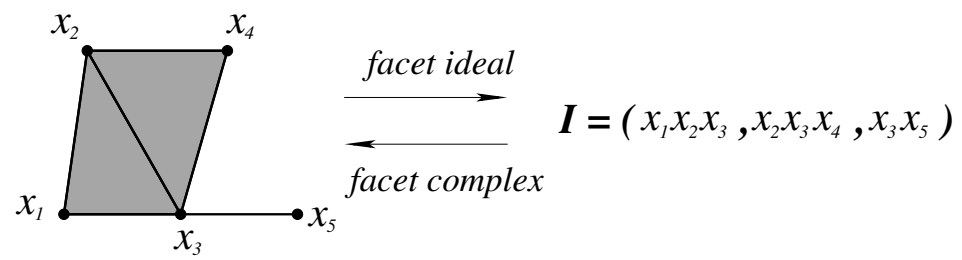

Figure 2: Facet ideal relationship

In [6], Fröberg shows that a square-free monomial ideal has a linear resolution if and only if the simplicial homology groups of its Stanley-Reisner complex and its induced subcomplexes vanish in all but one dimension.

Theorem 3 (Fröberg [6]). A square-free monomial ideal I has a t-linear resolution over a field $k$ if and only if $\tilde{H}_{i}\left((\mathcal{N}(I))_{S} ; k\right)=0$ for all $S \subseteq V(\mathcal{N}(I))$ and $i \neq t-2$.

Therefore one way to discover which square-free monomial ideals have linear resolutions is to examine the simplicial homology of their Stanley-Reisner complexes. In [2] it is shown that non-vanishing $d$-dimensional simplicial homology in characteristic 2 is equivalent to the presence of a particular combinatorial structure in the simplicial complex called a $\boldsymbol{d}$-dimensional cycle.

Definition 4 (d-dimensional cycle). For $d \geqslant 1$, a $\boldsymbol{d}$-dimensional cycle is a pure $d$-dimensional simplicial complex which is $d$-path-connected and has the property that each of its $(d-1)$-dimensional faces is contained in an even number of its $d$-dimensional faces.

Examples of 2-dimensional cycles are given in Figure 3.

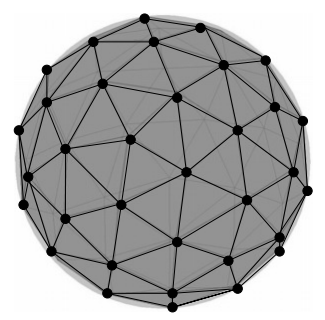

(a) A triangulation of the sphere

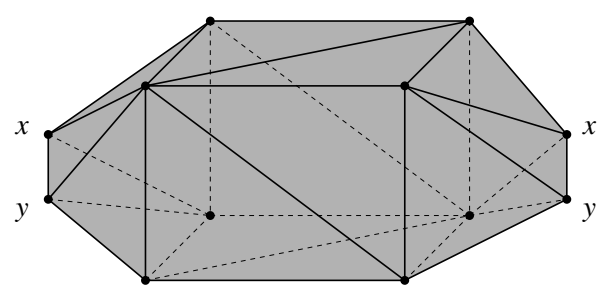

(b) A triangulation of the sphere pinched along a 1-face

Figure 3: Examples of 2-dimensional cycles. 
A graph cycle can be thought of as the 1-dimensional simplicial complex necessary and sufficient to generate 1-dimensional simplicial homology. The motivation for the concept of the $d$-dimensional cycle is to generalize the graph cycle to higher dimensions from this homological perspective. Recall that the support complex of a homological $d$-chain $c=\alpha_{1} F_{1}+\cdots+\alpha_{q} F_{q}$, where each $\alpha_{i}$ is a non-zero element of the field $k$ under consideration, is the simplicial complex $\left\langle F_{1}, \ldots, F_{q}\right\rangle$. The $d$-chain $c$ is a homological $\boldsymbol{d}$-cycle if it belongs to the kernel of the $d$-boundary operator so that $\partial_{d}(c)=0$. It is considered a $\boldsymbol{d}$-boundary if $\partial_{d+1}\left(c^{\prime}\right)=c$ for some $(d+1)$-chain $c^{\prime}$. In this paper we will make use of the following close relationship between $d$-dimensional cycles and homological $d$-cycles over the field $\mathbb{Z}_{2}$.

Proposition 5 (Proposition 5.1 in Connon [2]). The sum of the d-faces of a d-dimensional cycle is a homological d-cycle over $\mathbb{Z}_{2}$ and, conversely, the d-path-connected components of the support complex of a homological d-cycle over $\mathbb{Z}_{2}$ are d-dimensional cycles.

The following two propositions provide ways of building higher and lower-dimensional cycles from a $d$-dimensional cycle.

Proposition 6 (Proposition 4.4 in Connon [2]). Let $\Omega$ be a d-dimensional cycle with $d$ faces $F_{1}, \ldots, F_{k}$ in a simplicial complex $\Gamma$. Suppose that there exist $(d+1)$-faces $A_{1}, \ldots, A_{\ell}$ in $\Gamma_{V(\Omega)}$ such that, over $\mathbb{Z}_{2}$ we have

$$
\partial_{d+1}\left(\sum_{i=1}^{\ell} A_{i}\right)=\sum_{j=1}^{k} F_{j}
$$

and for no strict subset of $\left\{A_{1}, \ldots, A_{\ell}\right\}$ does (1) hold. Let $v$ be a vertex with $v \notin V(\Omega)$ and let $\Phi=\left\langle F_{1} \cup v, \ldots, F_{k} \cup v, A_{1}, \ldots, A_{\ell}\right\rangle$ then $\Phi$ is a $(d+1)$-dimensional cycle.

Proposition 7 (Proposition 4.3 in Connon [2]). Let $\Omega$ be a d-dimensional cycle and let $v \in V(\Omega)$. If $F_{1}, \ldots, F_{k}$ are the d-faces of $\Omega$ which contain $v$ then the $(d-1)$-pathconnected components of the complex $\left\langle F_{1} \backslash\{v\}, \ldots, F_{k} \backslash\{v\}\right\rangle$ are $(d-1)$-dimensional cycles.

A $d$-dimensional cycle is called face-minimal if no strict non-empty subset of its $d$-dimensional faces also forms a $d$-dimensional cycle. The complex in Figure $4 \mathrm{a}$ is an example of a non-face-minimal 2-dimensional cycle and the complex in Figure $4 \mathrm{~b}$ is a face-minimal 2-dimensional cycle.

Remark 8. It is not difficult to show that any $d$-dimensional cycle can be written as a disjoint union of face-minimal cycles (see [2, Lemma 4.7]).

To generalize Fröberg's criterion, we develop a higher-dimensional counterpart to chordal graphs.

Definition 9 (chord set, $\boldsymbol{d}$-chorded, chorded [3]). Given a $d$-dimensional cycle $\Omega$ in a simplicial complex $\Gamma$ a chord set of $\Omega$ in $\Gamma$ is a set $C$ of $d$-dimensional faces in $\Gamma_{V(\Omega)}$ not belonging to $\Omega$ which satisfy the following properties: 


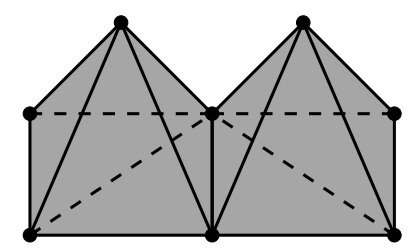

(a) Two hollow triangulated square pyramids glued along a 1-face

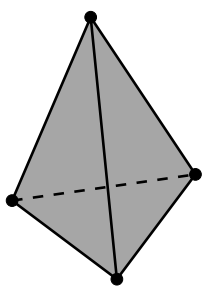

(b) The hollow tetrahedron

Figure 4: Non-face-minimal versus face-minimal

1. the simplicial complex whose set of facets is $C \cup$ Facets $(\Omega)$ consists of $k d$-dimensional cycles $\Omega_{1}, \ldots, \Omega_{k}$ for $k \geqslant 2$

2. each $d$-face in $C$ is contained in an even number of the cycles $\Omega_{1}, \ldots, \Omega_{k}$,

3. each $d$-face of $\Omega$ is contained in an odd number of the cycles $\Omega_{1}, \ldots, \Omega_{k}$,

4. $V\left(\Omega_{i}\right) \subsetneq V(\Omega)$ for $i=1, \ldots, k$.

A simplicial complex $\Gamma$ is $\boldsymbol{d}$-chorded if it is pure of dimension $d \geqslant 1$ and all faceminimal $d$-dimensional cycles in $\Gamma$ which are not $d$-complete have a chord set in $\Gamma$. We say that an arbitrary simplicial complex $\Gamma$ is chorded if $\Gamma^{[d]}$ is $d$-chorded for all $1 \leqslant d \leqslant \operatorname{dim} \Gamma$.

Remark 10. Notice that the conditions in Definition 9 imply that over $\mathbb{Z}_{2}$ we have $\sum_{i} \Omega_{i}=$ $\Omega$.

As a consequence of the properties of a chord set and Remark 8 all face-minimal $d$ dimensional cycles in a $d$-chorded complex can be broken down into cycles on fewer and fewer vertices until only $d$-complete cycles remain. It is shown in [2] that these are the $d$-dimensional cycles on the smallest number of vertices, namely $d+2$. The notion of a $d$-chorded simplicial complex generalizes the graph theoretic notion of a chordal graph. In particular a 1-chorded complex is a chordal graph and conversely. See Figure 5 for an example of a 2-chorded simplicial complex. This complex is comprised of a 2-dimensional cycle, the hollow octahedron, with a chord set shown in a darker shading.

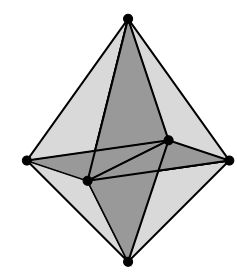

Figure 5: A 2-chorded simplicial complex

Some of the simplest examples of $d$-chorded simplicial complexes are the $d$-complete complexes. 
Proposition 11 (d-complete $\Rightarrow \boldsymbol{d}$-chorded). For $n \geqslant d+1$, the $d$-dimensional $d$ complete simplicial complex $\Lambda_{n}^{d}$ is d-chorded.

Proof. Let $\Omega$ be a face-minimal $d$-dimensional cycle in $\Lambda_{n}^{d}$ which is not $d$-complete. Then $\Omega$ is also a face-minimal $d$-dimensional cycle in the simplex on $V(\Omega)$. Call this simplex $\Gamma$. Since any simplex is acyclic over any field, we have $\tilde{H}_{d}\left(\Gamma ; \mathbb{Z}_{2}\right)=0$. Hence $\Omega$, which is the support complex of a $d$-cycle by Proposition 5 , is the support complex of a $d$-boundary over $\mathbb{Z}_{2}$. It is shown in [3, Lemma 5.9] that under these conditions $\Omega$ has a chord set in $\Gamma$. Since $\Lambda_{n}^{d}=\Gamma^{[d]}, \Omega$ has a chord set in $\Lambda_{n}^{d}$ as well. Therefore $\Lambda_{n}^{d}$ is $d$-chorded.

Recall that the clique complex of a graph $G$, denoted $\Delta(G)$, is the simplicial complex on the same vertex set as $G$ whose facets are given by the vertices in the maximal complete subgraphs of $G$. In fact, Fröberg originally gave Theorem 1 in terms of the Stanley-Reisner ideal of the clique complex of a graph. It is not hard to see that this ideal is equivalent to the edge ideal of a graph's complement.

Theorem 12 (Fröberg [7]). If a graph $G$ is chordal then the Stanley-Reisner ideal of $\Delta(G)$ has a 2-linear resolution over any field. Conversely, if the Stanley-Reisner ideal of a simplicial complex $\Gamma$ has a 2-linear resolution over any field, then $\Gamma=\Delta\left(\Gamma^{[1]}\right)$ and $\Gamma^{[1]}$ is chordal.

There exists a similar notion to the clique complex in higher dimensions.

Definition 13 ( $\boldsymbol{d}$-closure). The $\boldsymbol{d}$-closure of a pure $d$-dimensional simplicial complex $\Gamma$, denoted $\Delta_{d}(\Gamma)$, is the simplicial complex on $V(\Gamma)$ whose faces are given in the following way:

- the $d$-faces of $\Delta_{d}(\Gamma)$ are exactly the $d$-faces of $\Gamma$

- all subsets of $V(\Gamma)$ with at most $d$ elements are faces of $\Delta_{d}(\Gamma)$

- a subset of $V(\Gamma)$ with more than $d+1$ elements is a face of $\Delta_{d}(\Gamma)$ if and only if all of its subsets of $d+1$ elements are faces of $\Gamma$.

The $d$-closure of $\Gamma$ is also called the complex of $\Gamma[5]$ and the clique complex of $\boldsymbol{\Gamma}[10]$. We use the term $d$-closure to keep track of the dimension at which the operation is applied. Note that the pure $d$-skeleton of $\Delta_{d}(\Gamma)$ is $\Gamma$. See Figure 6 for an example of 2-closure.

The motivation for properties 2 and 3 in Definition 13 is algebraic in nature. We are interested in when the Stanley-Reisner ideals of these $d$-closures have $(d+1)$-linear resolutions. The minimal generators of these ideals must all have size $d+1$ and so the minimal non-faces of these complexes must all have size $d+1$. Therefore such a complex must contain all faces of dimension less than $d$ which is ensured by property 2 . As well, any non-face of size larger than $d+2$ must contain a minimal non-face of size $d+1$. This 


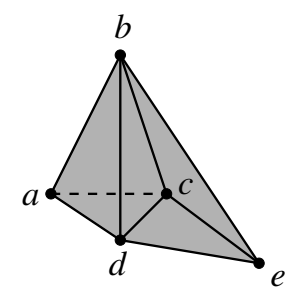

(a) $\Gamma=\langle a b c, a b d, a c d, b c d, b c e, c d e\rangle$

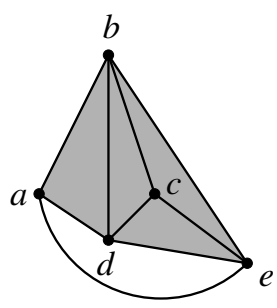

(b) $\Delta_{2}(\Gamma)=\langle a b c d, b c e, c d e, a e\rangle$

Figure 6: 2-closure

follows from property 3. Therefore the minimal generators of the Stanley-Reisner ideal of the $d$-closure of a simplicial complex will all have degree $d+1$.

The following lemma explains the results of subsequent applications of the closure operation on different dimensions.

Lemma 14 (The $n$-closure is stronger than the $m$-closure when $n<m$ ). Let $\Gamma$ be a pure $n$-dimensional simplicial complex.

1. If $m<n$ then $\Delta_{m}\left(\Delta_{n}(\Gamma)^{[m]}\right)$ is a simplex.

2. If $m=n$ then $\Delta_{m}\left(\Delta_{n}(\Gamma)^{[m]}\right)=\Delta_{n}(\Gamma)$.

3. If $m>n$ then $\Delta_{m}\left(\Delta_{n}(\Gamma)^{[m]}\right)^{[t]}=\Delta_{n}(\Gamma)^{[t]}$ for all $t \geqslant m$.

Proof.

1. If $m<n$ then $\Delta_{n}(\Gamma)^{[m]}$ is $m$-complete as the $n$-closure adds all faces of dimension less than $n$. Therefore by the definition of $m$-closure the set of all vertices of $\Delta_{n}(\Gamma)^{[m]}$ is a face of $\Delta_{m}\left(\Delta_{n}(\Gamma)^{[m]}\right)$ and so $\Delta_{m}\left(\Delta_{n}(\Gamma)^{[m]}\right)$ is a simplex.

2. If $m=n$ then by the nature of $n$-closure

$$
\Delta_{m}\left(\Delta_{n}(\Gamma)^{[m]}\right)=\Delta_{n}\left(\Delta_{n}(\Gamma)^{[n]}\right)=\Delta_{n}(\Gamma) .
$$

3. Let $m>n$ and let $F$ be a facet of $\Delta_{n}(\Gamma)^{[t]}$. Then every subset $A$ of $F$ of size $m+1 \leqslant$ $t+1$ is also a face of $\Delta_{n}(\Gamma)^{[t]}$ so $A \in \Delta_{n}(\Gamma)^{[m]}$. Therefore $F \in \Delta_{m}\left(\Delta_{n}(\Gamma)^{[m]}\right)^{[t]}$.

Conversely, if $F$ is a facet of $\Delta_{m}\left(\Delta_{n}(\Gamma)^{[m]}\right)^{[t]}$ then all subsets of $F$ of size $m+1 \leqslant t+1$ belong to $\Delta_{n}(\Gamma)^{[m]}$. Therefore $\Delta_{n}(\Gamma)_{F}^{[m]}$ is $m$-complete. Thus all subsets of $F$ of size $n+1<m+1$ are in $\Delta_{n}(\Gamma)^{[m]}$ which means they are $n$-faces of $\Gamma$. Hence by the definition of $n$-closure $F \in \Delta_{n}(\Gamma)^{[m]}$. 
In [3] we were able to show the following theorem which gives a necessary combinatorial condition for a Stanley-Reisner ideal to have a linear resolution over fields of characteristic 2. It is a generalization of one direction of Theorem 12 in the case of fields having characteristic 2 .

Theorem 15 (Theorem 6.1 in Connon and Faridi [3]). Let $\Gamma$ be a simplicial complex, let $k$ be any field of characteristic 2 and let $d \geqslant 1$. If $\mathcal{N}(\Gamma)$ has a $(d+1)$-linear resolution over $k$ then $\Gamma=\Delta_{d}\left(\Gamma^{[d]}\right)$ and $\Gamma^{[d]}$ is d-chorded.

The converse of Theorem 15 does not hold. The following is a counterexample.

Example 16. Let $\Gamma$ be the pure 2-dimensional simplicial complex on the vertex set $\left\{x_{0}, \ldots, x_{5}\right\}$ whose minimal non-faces are $\left\{x_{0}, x_{1}, x_{2}\right\}$ and $\left\{x_{3}, x_{4}, x_{5}\right\}$. Note that $\Gamma$ is the join of two triangle boundaries, and is thus a triangulation of a 3 -sphere. The complex $\Gamma$ is a 2-chorded simplicial complex and the Stanley-Reisner ideal of the 3-dimensional simplicial complex $\Delta_{2}(\Gamma)$ does not have a linear resolution over $\mathbb{Z}_{2}$. The pure 3 -skeleton of $\Delta_{2}(\Gamma)$ is a 3 -dimensional cycle with no chord set which is not 3-complete and we have $\tilde{H}_{3}\left(\Delta_{2}(\Gamma) ; \mathbb{Z}_{2}\right) \neq 0$.

In the next section we determine which $d$-chorded complexes have $d$-closures which do not have $(d+1)$-linear resolutions in characteristic 2. By doing this we give a necessary and sufficient combinatorial condition for an ideal to have a linear resolution over a field of characteristic 2 .

\section{Combinatorial criterion for linear resolution in characteristic 2}

As we can see from Theorem 3 for a square-free monomial ideal to have a linear resolution its Stanley-Reisner complex must have vanishing simplicial homology in all but one dimension. Theorem 15 shows that in characteristic 2 this corresponds to a pure complex that is $d$-chorded, where $d$ is the dimension of the complex.

Conversely, in order to show that a particular class of simplicial complexes have Stanley-Reisner ideals with linear resolutions we must show that the simplicial homology of these simplicial complexes and of their induced subcomplexes vanishes in the right dimensions. Consider any pure $d$-dimensional simplicial complex $\Gamma$. We know that $\Delta_{d}(\Gamma)$ contains all possible faces of dimension less than $d$. This means that $\Delta_{d}(\Gamma)$ cannot have any non-zero simplicial homology in dimensions less than $d-1$. Furthermore, when $\Gamma$ is $d$-chorded $\Delta_{d}(\Gamma)$ has vanishing homology in dimension $d$ also and so altogether we have the following result.

Proposition 17 (Proposition 5.8 in Connon and Faridi [3]). For any d-chorded simplicial complex $\Gamma$ and any field $k$ of characteristic 2 we have $\tilde{H}_{i}\left(\Delta_{d}(\Gamma)_{W} ; k\right)=0$ for all $W \subseteq$ $V(\Gamma), 0 \leqslant i \leqslant d-2$ and $i=d$.

As we can see from Example 16 it is not necessarily the case that the upper-level homology groups of the $d$-closure of a $d$-chorded complex vanish. In examples such as 
this the Stanley-Reisner ideal of the $d$-closure will not have a linear resolution. In these cases the $d$-closure of the complex has a pure $m$-skeleton which is not $m$-chorded for some $m>d$. When we require these $m$-skeletons to be $m$-chorded we obtain a necessary and sufficient condition for linear resolution over fields of characteristic 2.

Theorem 18 (Criterion for a linear resolution I). Let I be generated by square-free monomials of degree $d+1$. The following are equivalent:

a) I has a linear resolution over some (equivalently any) field of characteristic 2.

b) $\mathcal{N}(I)$ is chorded.

c) $\mathcal{N}(I)^{[m]}$ is $m$-chorded for all $m \geqslant d$.

d) $\Delta_{d}\left(\overline{\mathcal{F}}(I)_{d}\right)$ is chorded.

e) $\Delta_{d}\left(\overline{\mathcal{F}(I)}_{d}\right)^{[m]}$ is $m$-chorded for all $m \geqslant d$.

Proof. Let $\Gamma=\mathcal{N}(I)$ and let $\Upsilon=\overline{\mathcal{F}(I)}_{d}$.

a) $\Rightarrow$ b) Suppose that $I$ has a $(d+1)$-linear resolution over any field of characteristic 2 . By Theorem 15 we know that $\Gamma=\Delta_{d}\left(\Gamma^{[d]}\right)$ and $\Gamma^{[d]}$ is $d$-chorded. We also know that $\Gamma$ is $m$-complete for all $m<d$ by the definition of $d$-closure. Therefore it follows from Proposition 11 that $\Gamma^{[m]}$ is $m$-chorded for $m<d$.

Let $m>d$ and let $\Omega$ be any face-minimal, non- $m$-complete $m$-dimensional cycle in $\Gamma^{[m]}$. By Proposition 5 we know that $\Omega$ is the support complex of a homological $m$-cycle over $\mathbb{Z}_{2}$. The ideal $I$ has a linear resolution over $\mathbb{Z}_{2}$ and so we know that $\tilde{H}_{m}\left(\Gamma_{V(\Omega)} ; \mathbb{Z}_{2}\right)=0$ by Theorem 3. Thus $\Omega$ is also the support complex of an $m$-boundary of faces of $\Gamma_{V(\Omega)}$ over $\mathbb{Z}_{2}$. It follows by $[3$, Lemma 5.9$]$ we know that $\Omega$ has a chord set in $\Gamma_{V(\Omega)}$. Hence $\Gamma^{[m]}$ is $m$-chorded. Therefore $\Gamma^{[m]}$ is $m$-chorded for all $1 \leqslant m \leqslant \operatorname{dim} \Gamma$ and so $\Gamma$ is chorded.

b) $\Rightarrow$ c) This is clear.

c) $\Rightarrow$ a) Suppose that $\Gamma^{[m]}$ is $m$-chorded for all $m \geqslant d$. Since $I$ is generated by square-free monomials of degree $d+1$ then by [3, Proposition 5.6] we know that its Stanley-Reisner complex $\Gamma$ satisfies $\Gamma=\Delta_{d}\left(\Gamma^{[d]}\right)$. Therefore by Proposition 17 we know that for all $W \subseteq V(\Gamma)$ we have $\tilde{H}_{i}\left(\Gamma_{W} ; k\right)=0$ for $0 \leqslant i \leqslant d-2$ and $i=d$.

Let $m>d$ and let $W \subseteq V(\Gamma)$. We would like to show that $\tilde{H}_{m}\left(\Gamma_{W} ; k\right)=0$. By assumption $\Gamma^{[m]}$ is $m$-chorded. Therefore by Proposition 17 we know that $\tilde{H}_{m}\left(\Delta_{m}\left(\Gamma^{[m]}\right)_{W} ; k\right)=0$. Furthermore, by Lemma 14 we have

$$
\Delta_{m}\left(\Gamma^{[m]}\right)^{[t]}=\Delta_{m}\left(\Delta_{d}\left(\Gamma^{[d]}\right)^{[m]}\right)^{[t]}=\Delta_{d}\left(\Gamma^{[d]}\right)^{[t]}
$$

for all $t \geqslant m$. Thus the $m$-faces and the $m+1$-faces of $\Delta_{m}\left(\Gamma^{[m]}\right)_{W}$ and $\Gamma_{W}=\Delta_{d}\left(\Gamma^{[d]}\right)_{W}$ are equivalent. Therefore we have

$$
\tilde{H}_{m}\left(\Gamma_{W} ; k\right)=\tilde{H}_{m}\left(\Delta_{m}\left(\Gamma^{[m]}\right)_{W} ; k\right)=0
$$


for all $m>d$. Consequently $\tilde{H}_{m}\left(\Gamma_{W} ; k\right)=0$ for all $m \neq d-1$. Hence $I$ has a $(d+1)$-linear resolution by Theorem 3 .

b) $\Leftrightarrow$ d) It is easy to see that the $d$-complement of $\mathcal{F}(I)$ is equal to the pure $d$-skeleton of $\mathcal{N}(I)=\Gamma$. Thus $\Upsilon=\Gamma^{[d]}$ and so $\Delta_{d}(\Upsilon)$ is chorded if and only $\Delta_{d}\left(\Gamma^{[d]}\right)=\mathcal{N}(I)$ is chorded.

c) $\Leftrightarrow$ e) As before, $\Upsilon=\Gamma^{[d]}$ and so $\Delta_{d}(\Upsilon)^{[m]}$ is $m$-chorded for all $m \geqslant d$ if and only $\Delta_{d}\left(\Gamma^{[d]}\right)^{[m]}$ is $m$-chorded for all $m \geqslant d$.

The condition for $(d+1)$-linear resolution in Theorem 18 requires checking that every non- $m$-complete, face minimal $m$-dimensional cycle in $\mathcal{N}(I)^{[m]}$ has a chord set for all $m \geqslant d$ which can be tedious. However our next result shows that in most cases assuming that $\mathcal{N}(I)^{[d]}$ is $d$-chorded suffices. The only possible obstruction to this implication is the presence of a 1-complete, face-minimal, $m$-dimensional cycle which is not $m$-complete and which has no chord set. Thus to check for a linear resolution one need only verify that $\mathcal{N}(I)^{[d]}$ is $d$-chorded and that any cycles of this special nature have chord sets. In general we expect these types of cycles to occur infrequently. For example, in the 2dimensional case we find that of the $2^{35} 2$-dimensional cycles on 8 vertices only $0.063 \%$ are face-minimal and 1-complete without being 2-complete. In the case of 9 vertices there are $2^{56} 2$-dimensional cycles and approximately $0.00023 \%$ of those cycles fall into this category. It seems likely that this percentage would continue to decrease as the number of vertices increased as requiring a complete 1-skeleton is more restrictive the more vertices are present. An example of a simplicial complex which is 2-chorded, but whose closure contains one of these problem cycles is given in Example 16. In this case $\Gamma$ is 2 -chorded but $\Delta_{2}(\Gamma)^{[3]}$ is not 3 -chorded. The simplicial complex $\Delta_{2}(\Gamma)^{[3]}$ is a faceminimal, 1-complete, 3-dimensional cycle which is not 3-complete and which has no chord set. The Stanley-Reisner ideal of the 3-dimensional simplicial complex $\Delta_{2}(\Gamma)$ does not have a linear resolution over $\mathbb{Z}_{2}$.

For an illustration of the technique used in the proof of the following theorem see Figure 7.

Theorem 19 (Chordedness can be transferred upwards in the closure). Let $\Gamma$ be a d-chorded simplicial complex. Then $\Delta_{d}(\Gamma)$ is chorded if and only if for all $m>d$ each 1-complete, face-minimal, non-m-complete $m$-dimensional cycle in $\Delta_{d}(\Gamma)$ has a chord set in $\Delta_{d}(\Gamma)$.

Proof. If $\Delta_{d}(\Gamma)$ is chorded then all face-minimal, non- $m$-complete $m$-dimensional cycles in $\Delta_{d}(\Gamma)$ have chord sets in $\Delta_{d}(\Gamma)$ for all $m$ by definition.

Now suppose that for all $m>d$ each 1-complete, face-minimal, non- $m$-complete $m$ dimensional cycle in $\Delta_{d}(\Gamma)$ has a chord set in $\Delta_{d}(\Gamma)$. We would like to show that $\Delta_{d}(\Gamma)$ is chorded. By the nature of the $d$-closure we know that $\Delta_{d}(\Gamma)$ is $t$-complete for all $t<d$. Thus $\Delta_{d}(\Gamma)^{[t]}$ is $t$-chorded for all $t<d$ by Proposition 11 . 


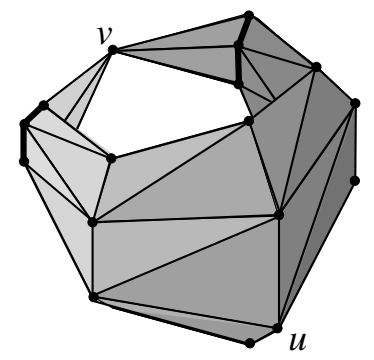

(a) The 2-dimensional cycle $\Omega$ with 1-dimensional cycles $\Phi_{1}$ and $\Phi_{2}$ shown in bold

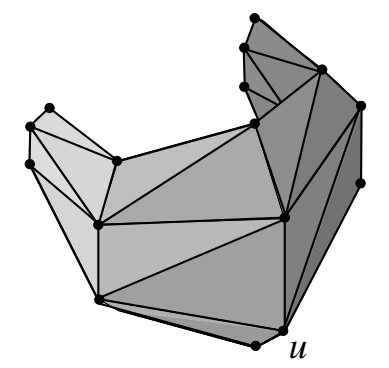

(c) The 2-dimensional cycle $\Omega_{3}=\left\langle H_{1}, \ldots, H_{s}\right\rangle$

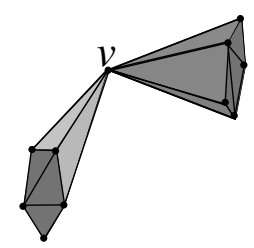

(b) The 2-dimensional cycles $\Omega_{1}$ and $\Omega_{2}$ joined by $v$

Figure 7: Construction used in the proof of Theorem 19.

For the remaining cases we will use induction on $t$. When $t=d$ we have $\Delta_{d}(\Gamma)^{[d]}=\Gamma$. Since $\Gamma$ is $d$-chorded by assumption this proves the base case.

Now suppose that $t>d$ and we know that $\Delta_{d}(\Gamma)^{[n]}$ is $n$-chorded for all $n<t$. Let $\Omega$ be a face-minimal $t$-dimensional cycle that is not $t$-complete in $\Delta_{d}(\Gamma)^{[t]}$. We would like to show that $\Omega$ has a chord set in $\Delta_{d}(\Gamma)^{[t]}$. If $\Omega$ is 1 -complete then by assumption $\Omega$ has a chord set in $\Delta_{d}(\Gamma)^{[t]}$, and so we may assume that $\Omega$ is not 1-complete. Then there exist $u, v \in V(\Omega)$ such that $u$ and $v$ are not contained in the same $t$-face of $\Omega$.

Let $F_{1}, \ldots, F_{k}$ be the $t$-faces of $\Omega$ containing $v$. By Proposition 7 we know that the $(t-1)$-path-connected components of $\left\langle F_{1} \backslash\{v\}, \ldots, F_{k} \backslash\{v\}\right\rangle$ are $(t-1)$-dimensional cycles. Call these cycles $\Phi_{1}, \ldots, \Phi_{m}$. For each $i \in\{1, \ldots, m\}$ let $P_{i} \subseteq\{1, \ldots, k\}$ be such that $F_{j} \backslash\{v\} \in \Phi_{i}$ if and only if $j \in P_{i}$. Since for each $j$ the face $F_{j} \backslash\{v\}$ must belong to exactly one of $\Phi_{1}, \ldots, \Phi_{m}$, the sets $P_{1}, \ldots, P_{m}$ form a partition of $\{1, \ldots, k\}$.

In [3, Lemma 5.7] it is shown that the sum of the $d$-faces of a $d$-dimensional cycle $\Theta$ in a $d$-chorded simplicial complex forms a $d$-boundary on $V(\Theta)$ in the $d$-closure of the complex over $\mathbb{Z}_{2}$. The complex $\Delta_{d}(\Gamma)^{[t-1]}$ is $(t-1)$-chorded by assumption and since, for each $i, \Phi_{i}$ is a $(t-1)$-dimensional cycle in $\Delta_{d}(\Gamma)^{[t-1]}$, the sum of the $(t-1)$-faces of $\Phi_{i}$ form a $(t-1)$-boundary in $\Delta_{t-1}\left(\Delta_{d}(\Gamma)^{[t-1]}\right)$ on $V\left(\Phi_{i}\right)$ over $\mathbb{Z}_{2}$. This also holds in $\Delta_{d}(\Gamma)$ by Lemma 14 .

Hence for each $i$ there exist $t$-faces $A_{1}^{i}, \ldots, A_{\ell_{i}}^{i}$ in $\Delta_{d}(\Gamma)_{V\left(\Phi_{i}\right)}$ such that 


$$
\partial_{t}\left(\sum_{j=1}^{\ell_{i}} A_{j}^{i}\right)=\sum\left((t-1) \text {-faces of } \Phi_{i}\right)
$$

Without loss of generality we may assume that the choice of $A_{1}^{i}, \ldots, A_{\ell_{i}}^{i}$ is minimal in the sense that for no strict subset of $A_{1}^{i}, \ldots, A_{\ell_{i}}^{i}$ is (2) satisfied. Let $\Omega_{i}$ be the simplicial complex whose facets are $\left\{F_{j} \mid j \in P_{i}\right\} \cup\left\{A_{1}^{i}, \ldots, A_{\ell_{i}}^{i}\right\}$. By Proposition 6, under these conditions, $\Omega_{i}$ is a $t$-dimensional cycle. Furthermore, $V\left(\Omega_{i}\right) \subsetneq V(\Omega)$ as $u \notin V\left(\Omega_{i}\right)$. Since $\Omega$ is a face-minimal $t$-dimensional cycle, each $\Omega_{i}$ must contain at least one $t$-face which is not in $\Omega$. We collect all of these $t$-faces in the non-empty set $C$ :

$$
C=\left\{A_{j}^{i} \notin \Omega \mid 1 \leqslant i \leqslant m, 1 \leqslant j \leqslant \ell_{i}\right\} .
$$

We would like to show that $C$ is a chord set of $\Omega$ in $\Delta_{d}(\Gamma)^{[t]}$.

Consider the collection of $t$-faces in $\Omega$ and those in $\Omega_{1}, \ldots, \Omega_{m}$ with repeats. Let $H_{1}, \ldots, H_{s}$ be the $t$-faces in this collection which appear an odd number of times so that over $\mathbb{Z}_{2}$ we have

$$
\sum_{i=1}^{s} H_{i}=\sum(t \text {-faces of } \Omega)+\sum_{i=1}^{m} \sum\left(t \text {-faces of } \Omega_{i}\right) .
$$

Since $\Omega$ and $\Omega_{1}, \ldots, \Omega_{m}$ are all $t$-dimensional cycles, by Proposition 5 they correspond to homological $t$-cycles over $\mathbb{Z}_{2}$. Therefore by (3) over $\mathbb{Z}_{2}$ we have,

$$
\partial_{t}\left(\sum_{i=1}^{s} H_{i}\right)=\partial_{t}\left(\sum(t \text {-faces of } \Omega)\right)+\sum_{i=1}^{m} \partial_{t}\left(\sum\left(t \text {-faces of } \Omega_{i}\right)\right)=0 \text {. }
$$

Hence the $t$-path-connected components of the simplicial complex $\left\langle H_{1}, \ldots, H_{s}\right\rangle$ are $t$-dimensional cycles by Proposition 5 . Call these cycles $\Omega_{m+1}, \ldots, \Omega_{M}$. We would like to show that our set $C$ is a chord set that breaks $\Omega$ into the cycles $\Omega_{1}, \ldots, \Omega_{M}$. By (3), after rearranging the sums, over $\mathbb{Z}_{2}$ we have

$$
\sum(t \text {-faces of } \Omega)=\sum_{i=1}^{M} \sum\left(t \text {-faces of } \Omega_{i}\right)
$$

By noticing that the set $C$ is exactly those $t$-faces on the right-hand side of this equation which do not belong to $\Omega$ we can see that properties 2 and 3 of a chord set hold for $C$. Also, it is clear from our construction that all $t$-faces of both $\Omega$ and of $C$ appear in at least one of the $\Omega_{i}$ 's. Therefore property 1 of a chord set holds for the set $C$.

Now since none of $\Omega_{1}, \ldots, \Omega_{m}$ contain $u$ by construction we have $\left|V\left(\Omega_{i}\right)\right|<|V(\Omega)|$ for all $1 \leqslant i \leqslant m$. We would like to show that none of $\Omega_{m+1}, \ldots, \Omega_{M}$ contain $v$. Recall that $\Phi_{1}, \ldots, \Phi_{m}$ are the $(t-1)$-path-connected components of $\left\langle F_{1} \backslash\{v\}, \ldots, F_{k} \backslash\{v\}\right\rangle$ and so no two such distinct components could share a face of the form $F_{i} \backslash\{v\}$. Thus each face $F_{i}$ appears in only one of the cycles $\Omega_{1}, \ldots, \Omega_{m}$. Each such $F_{i}$ is also a face of $\Omega$ and so by our choice of $H_{1}, \ldots, H_{s}$ we know that we cannot have $F_{i}=H_{j}$ for any $i \in\{1, \ldots, k\}$ and 
$j \in\{1, \ldots, s\}$. Therefore, by the construction of the cycles $\Omega_{m+1}, \ldots, \Omega_{M}$ we know that none of the $F_{i}$ 's appear in any of these cycles. Recall that $F_{1}, \ldots, F_{k}$ are the only $t$-faces of $\Omega$ that contain $v$ and none of the $t$-faces of $C$ contain $v$ since they are subsets of $\bigcup_{i=1}^{m} V\left(\Phi_{i}\right)$. It follows that none of $\Omega_{m+1}, \ldots, \Omega_{M}$ contain $v$. This implies that $\left|V\left(\Omega_{i}\right)\right|<|V(\Omega)|$ for all $m+1 \leqslant i \leqslant M$. Thus property 4 of a chord set is also satisfied by $C$ and hence $\Delta_{d}(\Gamma)^{[t]}$ is $t$-chorded. Hence $\Delta_{d}(\Gamma)$ is chorded.

As a consequence of Theorems 18 and 19 we have the following theorem.

Theorem 20 (Criterion for a linear resolution II). Let I be generated by square-free monomials of degree $d+1$. Then I has a linear resolution over any (equivalently, some) field of characteristic 2 if and only if $\mathcal{N}(I)^{[d]}$ is $d$-chorded and for $m>d$ each 1-complete, face-minimal, non-m-complete $m$-dimensional cycle in $\mathcal{N}(I)$ has a chord set in $\mathcal{N}(I)$.

We close by noting that in the 1-dimensional case, such obstructions to linear resolution do not exist. In particular if $\Gamma^{[1]}$ is 1-chorded then in $\Delta_{1}\left(\Gamma^{[1]}\right)$ all 1-complete $m$-dimensional cycles lie in $m$-complete induced subcomplexes which are $m$-chorded and consequently such cycles have chord sets.

\section{Acknowledgements}

The authors would like to thank Rashid Zaare-Nahandi whose comments inspired the writing of this paper, and MSRI for their hospitality during the preparation of this paper. The authors would also like to thank the referee for their careful reading of the paper.

\section{References}

[1] E. Connon. Generalizing Fröberg's theorem on ideals with linear resolutions. PhD thesis, Dalhousie University, 2013.

[2] E. Connon. On $d$-dimensional cycles and the vanishing of simplicial homology. arXiv:1211.7087v2, 2013.

[3] E. Connon and S. Faridi. Chorded complexes and a necessary condition for a monomial ideal to have a linear resolution. J. Comb. Theory Ser. A, 120:1714-1731, 2013.

[4] J. Eagon and V. Reiner. Resolutions of Stanley-Reisner rings and Alexander duality. J. Pure Appl. Algebra, 130(3):265-275, 1998.

[5] E. Emtander. A class of hypergraphs that generalizes chordal graphs. Math. Scand., 106(1):50-66, 2010.

[6] R. Fröberg. Rings with monomial relations having linear resolutions. J. Pure Appl. Algebra, 38:235-241, 1985.

[7] R. Fröberg. On Stanley-Reisner rings. In Topics in Algebra, Part II, volume 26, pages 57-70, Warsaw, 1990. Banach Center Publ., PWN. 
[8] H. T. Hà and A. Van Tuyl. Monomial ideals, edge ideals of hypergraphs, and their graded Betti numbers. J. Algebraic Combin., 27(2):215-245, 2008.

[9] M. Morales, A. Nasrollah Nejad, A. A. Yazdan Pour, and R. Zaare-Nahandi. Monomial ideals with 3-linear resolutions. 2012. arXiv:1207.1789v1.

[10] M. Morales, A. A. Yazdan Pour, and R. Zaare-Nahandi. Regularity and free resolution of ideals which are minimal to $d$-linearity. 2012. arXiv:1207.1790v1.

[11] R. Woodroofe. Chordal and sequentially Cohen-Macaulay clutters. Electron. J. Combin., 18(1):\#P208, 2011. 